\title{
Gene therapy for type 1 diabetes: a novel approach for targeted treatment of autoimmunity
}

\author{
Rémi J. Creusot and C. Garrison Fathman \\ Department of Medicine, Division of Immunology and Rheumatology, Stanford University School of Medicine, Stanford, California, USA.
}

\begin{abstract}
It has been difficult to develop therapies that target those $T$ cells initiating and mediating the pathogenesis of autoimmune disease. Indeed, most current treatments indiscriminately affect both the autoreactive $T$ cells and the "good" $T$ cells, putting the patient at risk of compromised immune function. A new approach (see the related article beginning on page 969) raises the possibility of targeted therapy for autoimmunity. Transplantation of hematopoietic stem cells modified to express a protective form of MHC class II corrects a defect in central tolerance. This method contrasts with other targeted therapies that attempt to modify peripheral tolerance, which is also defective in type 1 diabetes mellitus.
\end{abstract}

\section{Type 1 diabetes is the result of several combined defects}

Type 1 diabetes mellitus (T1DM) results from $T$ cell-mediated autoimmune destruction of pancreatic insulin-producing $\beta$ cells. In the nonobese diabetic (NOD) mouse model of T1DM, multiple defects have been reported. The most striking (and perhaps clinically relevant) of these defects is a polymorphism in the $\beta$ chain of the MHC class II complex (the NOD variant is known as I-A $\left.\beta^{g 7}\right)$. Indeed, $\beta$ chains from "at-risk" alleles have at least 1 amino acid substitution, which leads to improper interactions with the $\alpha$ chain and aberrant peptide presentation. Such conformational changes in the MHC/peptide complex may result in altered thymic selection (inefficient negative selection [deletion] of self-antigen-specific $T$ cells or possibly inefficient positive selection [retention] of potential regulatory $\mathrm{T}$ cells [Treg's] that could control T1DM) $(1,2)$. Another defect in the NOD thymus affecting $T$ cell apoptosis was later described as being I-A $\beta^{g 7}$ independent (3). The autoreactive $T$ cells that escape the checkpoint of central tolerance need to be controlled in the periphery, by either tolerogenic DCs, Treg's, or both. However, it appears that both cell types are somehow inefficient at ensuring peripheral tolerance in NOD mice. The ratio between different subsets of DCs

Nonstandard abbreviations used: HSC, hematopoietic stem cell; NOD, nonobese diabetic; T1DM, type 1 diabetes mellitus; Treg, regulatory $\mathrm{T}$ cell.

Conflict of interest: The authors have declared that no conflict of interest exists.

Citation for this article: J. Clin. Invest. 114:892-894 (2004). doi:10.1172/JCI200423168. is altered in these mice, and so is their function (4). Furthermore, potentially tolerogenic DCs are functionally impaired due to their inability to induce tryptophan catabolism in response to IFN- $\gamma$ (5), while IL-12 production by potentially immunogenic DCs is increased following their activation as a result of a reduced ability to downregulate NF-кB (6), which may contribute to the autoimmune phenomenon. Although the number of Treg's in these mice was reported to be somewhat low (7), this observation has been more recently questioned (8). Susceptibility to the disease in NOD mice is also associated with a polymorphism in CTLA-4 (9), a surface marker that plays a role in the function of Treg's and in tolerance in general. In human patients, several defects affecting Treg's have also been reported (10): reduced numbers of $\mathrm{CD} 4{ }^{+} \mathrm{CD} 25^{+} \mathrm{T}$ cells and deficient cytokine production by $\mathrm{V} \alpha 24^{+} \mathrm{NK} T$ cells, the equivalent of the murine regulatory $\mathrm{V} \alpha 14^{+} \mathrm{NK} \mathrm{T}$ cell population. Human T1DM is also associated with polymorphisms in MHC class II and CTLA-4 $(1,7)$. Thus, the maintenance of tolerance is affected at both the central and the peripheral levels (Figure 1A). Here, we comment on several approaches that may efficiently correct one or the other tolerance mechanism and have the advantage of doing so by specifically targeting autoreactive $\mathrm{T}$ cells without dampening the general immune response.

\section{Gene therapy to restore central tolerance}

In this issue of the JCI, Tian and colleagues (11) describe a remarkable way to reestablish central tolerance in NOD mice through reconstitution with hematopoietic stem cells (HSCs) retrovirally transduced to express a protective form of the MHC class II $\beta$ chain. These studies were based on the previous observations that transgenic expression of such protective MHC class II molecules in NOD mice completely abrogates the development of diabetes $(12,13)$. Here, the investigators achieve coexpression of protective $\beta$ chains $\left(\mathrm{I}-\mathrm{A} \beta^{\mathrm{d}}\right.$ or I-A $\left.\beta^{\mathrm{k}}\right)$ with the endogenous disease-prone $\beta$ chain (I-A $\beta^{\mathrm{g} 7}$ ) in about $15 \%$ of bone marrow-derived HSCs; however this is sufficient to block disease progression in all irradiated/reconstituted NOD recipients (11). The transplanted mice are also resistant to cyclophosphamide-induced diabetes (a treatment known to accelerate the disease). A fraction of cells, expressing the retrovirally encoded $\beta$ chain in the cytoplasm (easily visualized because it is fused with GFP), is observed in each of the T cell, B cell, DC, and macrophage populations. However, it is interesting that only the cell types normally able to present the endogenous $\beta$ chain on their surface (i.e., antigen-presenting cells) are capable of cell-surface expression of the retrovirally encoded $\beta$ chain. Mice transplanted with forms of MHC class II that are protective against the disease exhibit no detectable leukocyte infiltration into the pancreatic islets and no cytokine (i.e., IFN- $\gamma$, IL-2, and IL-4) production by splenocytes in response to GAD65 and insulin, two putative autoantigens of T1DM. The investigators further show that a population of autoreactive $T$ cells normally present in the thymus of NOD mice, identifiable by tetramer staining, is virtually absent in the mice treated to express I-A $\beta^{d}$. On closer inspection, a few tetramer-positive cells are detectable in the double-positive, $\mathrm{CD}^{+}{ }^{+} \mathrm{CD} 8^{+} \mathrm{T}$ cell subset in the thymus of treated mice, but none of them succeed in further maturing into single-positive, $\mathrm{CD}^{+} \mathrm{CD}^{-} \mathrm{T}$ cells, which suggests that these potentially autoreactive $\mathrm{T}$ cells are effectively deleted in the thymus by nega- 


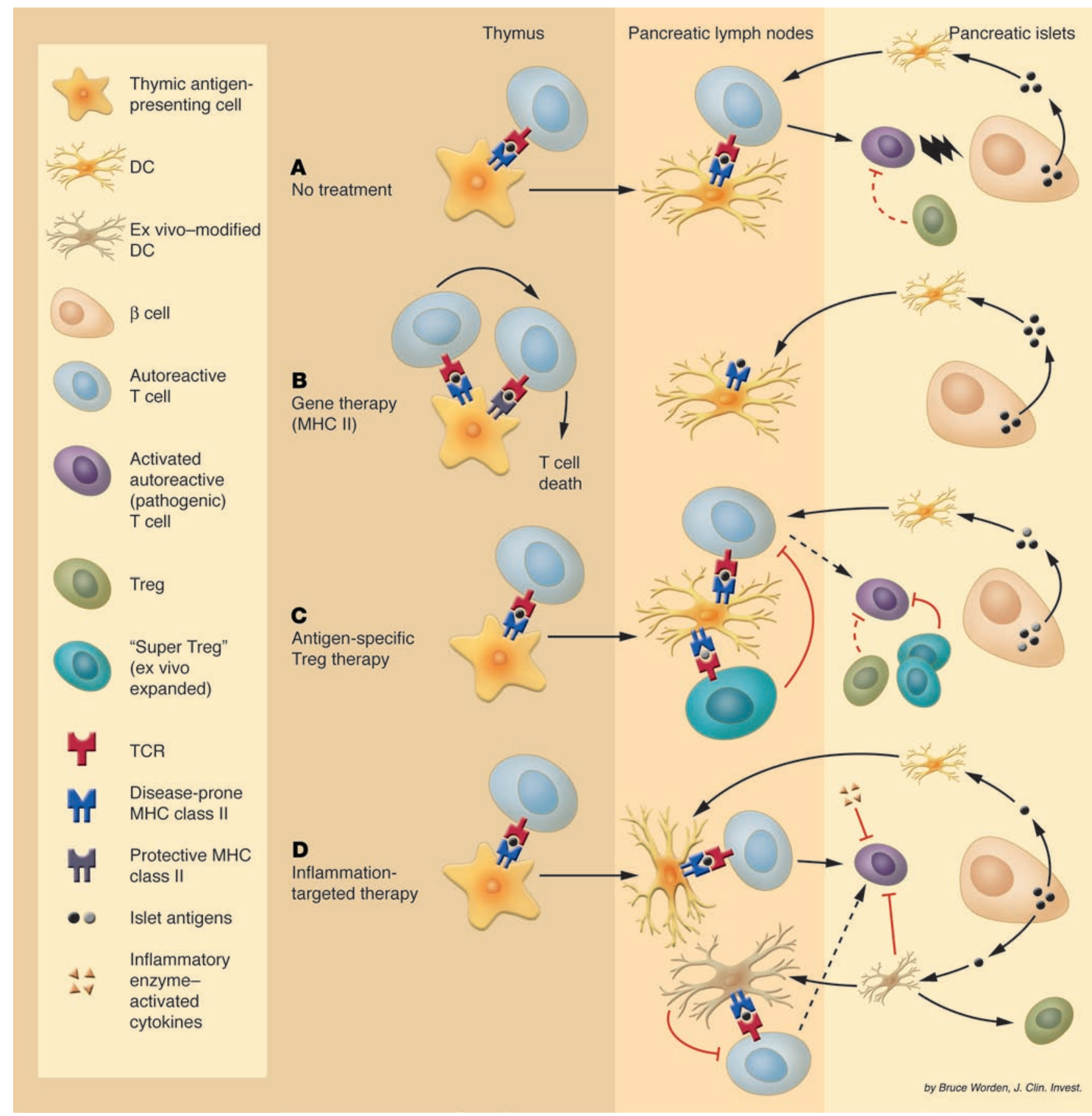

Figure 1

(A) Due to several combined defects, autoreactive T cells in NOD mice escape negative selection in the thymus and become activated in the pancreatic lymph nodes. The resulting effector T cells are kept under control for a limited period (resulting in peri-insulitis) until regulation (likely to involve Treg's) is no longer sufficient, and overt T1DM develops. (B) In irradiated NOD mice reconstituted with HSCs expressing a protective form of MHC class II, autoreactive T cells are efficiently deleted in the thymus and absolutely no insulitis is observed. (C) Antigen-specific Treg's from the islet infiltrate may be isolated and expanded ex vivo. When reintroduced, these cells prove to be efficient suppressors and may do so both in the pancreatic lymph nodes and in the pancreatic lesions. (D) DCs (or antigen-specific T cells) may be isolated and modified ex vivo to express: (a) immunoregulatory proteins that induce tolerance, apoptosis, or immune deviation of autoreactive T cells; and (b) appropriate receptors to enhance their trafficking into the sites of inflammation. Alternatively, inhibitory cytokines that have been engineered to become activated only under inflammatory conditions can be injected. These two strategies favor local versus systemic action of inhibitory products. 
tive selection (Figure 1B). Among the current strategies investigated for the treatment of T1DM and other autoimmune diseases, the gene therapy approach proposed by Tian and colleagues (11) could be one of the most difficult to apply to humans, but if this is possible, it might ensure long-lasting protection against the development of T1DM.

\section{The use of antigen-specific Treg's}

Despite several reports suggesting reduced numbers of $\mathrm{CD} 4^{+} \mathrm{CD} 25^{+}$Treg's in diabetic individuals $(7,10)$, an active subpopulation of Treg's, expressing high levels of IL-10 and inducible costimulatory molecules, was detected in the islets of prediabetic NOD mice (14). Like their pathogenic counterpart, these Treg's may also exhibit specificity toward one or several islet antigens. In two recent concurrent reports, researchers have isolated such antigen-specific Treg's and expanded them ex vivo using either DCs (15) or a combination of anti-CD3, anti-CD28, and IL-2 (16). These expanded Treg's were better suppressors than those freshly isolated in vivo and, unlike polyclonal Treg's, efficiently prevented the onset of overt disease upon adoptive transfer in prediabetic NOD mice (Figure 1C). In addition, antigen-specific Treg's could even prevent insulitis when injected in young mice (15) and exerted their effect up to 2 weeks after overt diabetes was diagnosed (15-16). These reports no doubt will result in intensive research efforts to isolate and expand Treg's from diabetic patients. The isolation step certainly poses challenges, as it is unclear whether antigen-specific Treg's circulate in the blood in sufficient numbers. Interestingly, it may be possible to isolate more cells from the whole pool of antigenspecific T cells, Treg's or not, and then convert them into Treg's by transduction with the gene expressing the transcription factor Foxp3 (17).

\section{Other strategies: inflammation- targeted therapies}

Many products have shown some efficacy in blocking the function of pathogenic
$\mathrm{T}$ cells in autoimmune diseases; these include, but are not limited to, inhibitory cytokines, antibodies or soluble receptors to proinflammatory cytokines, and immunosuppressive drugs. To avoid affecting the whole immune system, it is important to confine the activity of cell-specific therapy to the inflamed lesions. Adoptive cellular gene therapy uses different cell types with specific migratory capabilities (that have also been expanded and transduced ex vivo) as vehicles to express immunoregulatory products locally, at sites of inflammation (18). On the one hand, antigen-specific $\mathrm{T}$ cells have proven useful vehicles in the mouse model of rheumatoid arthritis (19), but their isolation from human patients is limiting. On the other hand, DCs are easier to isolate from the blood as precursors, and they can then be differentiated and transduced ex vivo. In the immature stage, they can migrate to sites of inflammation, and this migration pattern may be enhanced or prolonged by transgenic expression of specific chemokine receptors. If suitably conditioned ex vivo, they may even retain their immature phenotype and be tolerogenic (Figure 1D). Finally, some drugs may be designed so that they mediate their effect only within the sites of inflammation, as illustrated by an engineered TGF- $\beta$ that remains in an inactive form until converted into the active form upon cleavage by enzymes secreted in inflamed lesions (20).

In conclusion, emerging approaches are proving that it may be possible one day to block an undesirable immune response in an antigen-specific or a site-specific manner. Among them, the study by Tian and colleagues (11) is pioneering research toward reinstating central tolerance, possibly the most effective way to cure autoimmunity.

Address correspondence to: C. Garrison Fathman, Department of Medicine, Division of Immunology and Rheumatology, Stanford University School of Medicine, 269 West Campus Drive, Stanford, California 94305, USA. Phone: (650) 723-7887; Fax: (650) 725-1958; E-mail: cfathman@standford.edu.
1. McDevitt, H.O. 1998. The role of MHC class II molecules in susceptibility and resistance to autoimmunity. Curr. Opin. Immunol. 10:677-681.

2. Ridgway, W.M., Fasso, M., and Fathman, C.G. 1999. A new look at MHC and autoimmune disease. Science. 284:749-751.

3. Kishimoto, H., and Sprent, J. 2001. A defect in central tolerance in NOD mice. Nat. Immunol. 2:1025-1031.

4. Vasquez, A.C., Feili-Hariri, M., Tan, R.J., and Morel, P.A. 2004. Qualitative and quantitative abnormalities in splenic dendritic cell populations in NOD mice. Clin. Exp. Immunol. 135:209-218.

5. Grohmann, U., et al. 2003. A defect in tryptophan catabolism impairs tolerance in nonobese diabetic mice. J. Exp. Med. 198:153-160.

6. Weaver, D.J., Jr., et al. 2001. Dendritic cells from nonobese diabetic mice exhibit a defect in NFkappa B regulation due to a hyperactive I kappa B kinase. J. Immunol. 167:1461-1468.

7. Wu, A.J., Hua, H., Munson, S.H., and McDevitt, H.O. 2002. Tumor necrosis factor-alpha regulation of CD4+CD25+ T cell levels in NOD mice. Proc. Natl. Acad. Sci. U. S. A. 99:12287-12292.

8. Berzins, S.P., Venanzi. E.S., Benoist, C., and Mathis, D. 2003. T-cell compartments of prediabetic NOD mice. Diabetes. 52:327-334.

9. Ueda, H., et al. 2003. Association of the T-cell regulatory gene CTLA4 with susceptibility to autoimmune disease. Nature. 423:506-511.

10. Kukreja, A., et al. 2002. Multiple immuno-regulatory defects in type-1 diabetes. J. Clin. Invest. 109:131-140. doi:10.1172/JCI200213605.

11. Tian, C., et al. 2004. Prevention of type 1 diabetes by gene therapy. J. Clin. Invest. 114:969-978. doi:10.1172/JCI200422103.

12. Slattery, R.M., et al. 1990. Prevention of diabetes in non-obese diabetic I-Ak transgenic mice. Nature. 345:724-726.

13. Lund, T., et al. 1990. Prevention of insulin-dependent diabetes mellitus in non-obese diabetic mice by transgenes encoding modified I-A beta-chain or normal I-E alpha-chain. Nature. 345:727-729.

14. Herman, A.E., Freeman, G.J., Mathis, D., and Benoist, C. 2004. CD4+CD25+ T regulatory cells dependent on ICOS promote regulation of effector cells in the prediabetic lesion. J. Exp. Med. 199:1479-1489.

15. Tarbell, K.V., Yamazaki, S., Olson, K., Toy, P., and Steinman, R.M. 2004. CD25+ CD4+ T cells, expanded with dendritic cells presenting a single autoantigenic peptide, suppress autoimmune diabetes. J. Exp. Med. 199:1467-1477.

16. Tang, Q., et al. 2004. In vitro-expanded antigenspecific regulatory $\mathrm{T}$ cells suppress autoimmune diabetes. J. Exp. Med. 199:1455-1465.

17. Hori, S., Nomura, T., and Sakaguchi, S. 2003. Control of regulatory $\mathrm{T}$ cell development by the transcription factor Foxp3. Science. 299:1057-1061.

18. Tarner, I.H., Muller-Ladner, U., and Fathman, C.G. 2004. Targeted gene therapy: frontiers in the development of 'smart drugs'. Trends Biotechnol. 22:304-310.

19. Nakajima, A., et al. 2001. Antigen-specific T cellmediated gene therapy in collagen-induced arthritis. J. Clin. Invest. 107:1293-1301.

20. Adams, G., Vessillier, S., Dreja, H., and Chernajovsky, Y. 2003. Targeting cytokines to inflammation sites. Nat. Biotechnol. 21:1314-1320. 